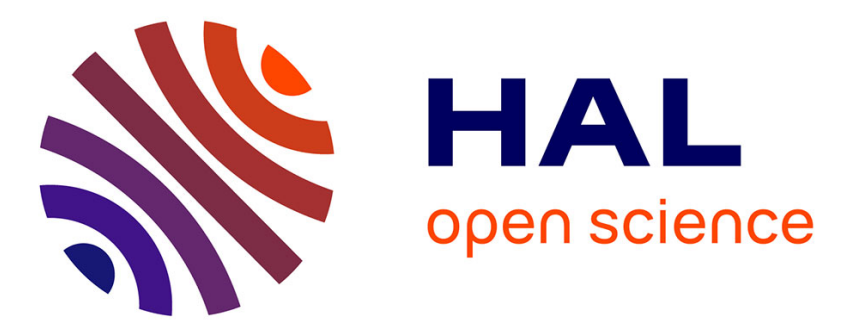

\title{
Internal gravity waves: parametric instability and deep ocean mixing
}

Chantal Staquet

\section{To cite this version:}

Chantal Staquet. Internal gravity waves: parametric instability and deep ocean mixing. Comptes Rendus Mécanique, 2007, 335 (9-10), pp.665-678. 10.1016/j.crme.2007.08.009 . hal-00265867

\section{HAL Id: hal-00265867 \\ https://hal.science/hal-00265867}

Submitted on 26 Apr 2020

HAL is a multi-disciplinary open access archive for the deposit and dissemination of scientific research documents, whether they are published or not. The documents may come from teaching and research institutions in France or abroad, or from public or private research centers.
L'archive ouverte pluridisciplinaire HAL, est destinée au dépôt et à la diffusion de documents scientifiques de niveau recherche, publiés ou non, émanant des établissements d'enseignement et de recherche français ou étrangers, des laboratoires publics ou privés. 


\title{
Internal gravity waves: parametric instability and deep ocean mixing
}

\author{
Chantal Staquet \\ Laboratoire des écoulements géophysiques et industriels, CNRS-INPG-UJF, B.P. 53, 38041 Grenoble cedex 9, France
}

Received 22 January 2007; accepted after revision 4 June 2007

\begin{abstract}
The Boussinesq approximation provides a convenient framework to describe the dynamics of stably-stratified fluids. A fundamental motion in these fluids consists of internal gravity waves, whatever the strength of the stratification. These waves may be unstable through parametric instability, which results in turbulence and mixing. After a brief review of the main properties of internal gravity waves, we show how the parametric instability of a monochromatic internal gravity wave organizes itself in space and time, using energetics arguments and a simple kinematic model. We provide an example, in the deep ocean, where such instability is likely to occur, as estimates of mixing from in situ measurements suggest. We eventually discuss the fundamental role of internal gravity wave mixing in the maintenance of the abyssal thermal stratification.
\end{abstract}

\section{Résumé}

Sur les ondes internes de gravité : de l'instabilité paramétrique au mélange abyssal dans l'océan. L'approximation de Boussinesq constitue un cadre bien adapté à l'étude des fluides stablement stratifiés. Des ondes de gravité internes s'y développent, quel que soit le niveau de stratification, qui peuvent être instables par instabilité paramétrique. Turbulence et mélange en résultent. Après un bref rappel sur les propriétés des ondes de gravité internes, nous montrons comment s'organisent les transferts d'énergie, dans l'espace et dans le temps, lorsqu'une onde interne est paramétriquement instable. Un modèle cinématique simple est employé pour cela. Puis nous illustrons ce processus et ses conséquences par un exemple océanique : l'instabilité paramétique se produit très certainement dans l'océan profond, comme le suggèrent les mesures in situ. Nous discutons finalement du rôle fondamen-tal des ondes de gravité internes dans l'entretien de la stratification abyssale, par le mélange qu'elles induisent.

Keywords: Fluid mechanics; Stably-stratified fluid; Internal gravity waves; Ocean mixing

Mots-clés : Mécanique des fluides; Fluide stratifié; Ondes de gravité internes ; Mélange océanique

\section{Introduction}

As discussed in classical textbooks of fluid mechanics (see [1]) and of geophysical fluid dynamics (see [2]), the Boussinesq approximation is an appropriate framework for describing the dynamics of stably-stratified fluids.

E-mail address: Chantal.Staquet@hmg.inpg.fr. 
A stably-stratified fluid is defined by the existence of a stable vertical density gradient, created by either a temperature or a concentration (e.g. salinity) gradient, which opposes any vertical motion. A stably-stratified fluid at rest satisfies hydrostatic equilibrium. In the ocean, the density fluctuations with respect to this equilibrium state are several orders of magnitude smaller than the mean value of the hydrostatic density field. These fluctuations do not influence inertia but give rise to a buoyancy force (namely, the sum of the weight and the Archimedean force). Typical frequencies are of the order of $10^{-3}-10^{-2} \mathrm{~s}^{-1}$ so that fluid motions are totally decoupled from sound waves and the fluid can be assumed to be incompressible. The Boussinesq approximation, consisting of the neglect of density variations in the inertia term, therefore provides a very convenient framework to describe internal gravity wave motions.

Motions in a stably-stratified fluid are fundamentally constrained by the buoyancy force, which is a restoring force. Adopting a fluid particle point of view, any fluid particle displaced vertically from its equilibrium position will be restored toward this position and, like a pendulum or a spring, will acquire an oscillatory motion. In a continuous fluid medium, internal gravity waves are generated.

Internal gravity waves are thus ubiquitous in geophysical fluids. In the stratosphere, which is the part of the atmosphere comprised between $\simeq 10$ and $\simeq 50 \mathrm{~km}$, velocity fluctuations are mostly contributed by internal gravity wave motions. These waves partly result from the blowing of the wind over the mountainous Earth surface, as their energy propagates nearly vertically upwards. In the ocean, the blowing of the wind on the sea surface mixes the ocean over one hundred meters or so (this is the so-called mixed layer); internal gravity waves are produced in the mixed layer and can propagate down into the deep ocean ([3]). By 'deep ocean', we mean the part of the ocean located below about $1000 \mathrm{~m}$ (that is, below the main thermocline, when present). In addition to the wind, the other main source of energy in the ocean is the tide, when interacting with the submarine topography.

Internal gravity waves are generated whatever the intensity of the background stratification and it is remarkable that they may be unstable whatever that intensity (provided the growth rate of the instability is large enough to overcome the stabilizing action of viscous effects). This was first shown from resonant interaction theory for infinitely small amplitude waves (see [4] for a review) and from stability analysis of a monochromatic internal gravity wave ([5-7]). Such a wave is most unstable to small scale perturbations (relative to its wavelength) and the instability is of the sub-harmonic parametric type (see [8] for a review). In other words, a monochromatic internal gravity wave transfers energy to small scale motions through parametric instability. Therefore this instability is one of the processes which lead to fluid mixing by internal gravity wave motions. The ability of internal gravity waves to mix stably-stratified fluids gives them a fundamental role in the deep ocean (cf. [9]).

These topics are successively addressed in the present article. In the next section, we briefly recall the main features of internal gravity waves. We show in Section 3 that the parametric instability of an internal gravity wave can be interpreted as an optimum scenario for extracting energy from the wave. Turbulence resulting from internal gravity wave breaking is briefly addressed in Section 4 . The oceanic context provides a framework for internal gravity generation, parametric instability and induced mixing, which is currently the subject of active research, and this is discussed in Section 5. Conclusions are proposed in the final section.

\section{Background}

\subsection{Dispersion relation of internal gravity waves}

Internal gravity waves are created by the buoyancy force (ignoring rotation effects), which is directed along the vertical. A direct consequence is that the dispersion relation of a monochromatic internal gravity wave is anisotropic, as we show now. The dispersion relation relates the wave frequency $\Omega$ to the wave vector $\mathbf{k}$. It can be computed from energetic considerations in a straightforward and simple manner, without dealing with the linearized equations of motions ([10]). According to Lighthill [1], the general expression of the dispersion relation is:

$$
\Omega^{2}=\frac{\text { generalized stiffness }}{\text { generalized inertia }}
$$

where the generalized stiffness is the coefficient of the squared displacement in the expression of the potential energy and the generalized inertia is the coefficient of the squared temporal derivative of the displacement in the expression of the kinetic energy.

The potential energy is the work done by the restoring force from the current position to the equilibrium position. An approximate expression of the buoyancy force, valid for small vertical displacements, can easily be inferred 
from the fluid particle view point we already adopted in the Introduction. When a fluid particle of unit volume is displaced vertically upward (say) from position $z_{0}$ to position $z_{0}+\zeta$ in a stably-stratified fluid with background density $\rho_{0}+\bar{\rho}(z)$, the buoyancy force is $g(\mathrm{~d} \bar{\rho} / \mathrm{d} z) \zeta$ (at first order in $\zeta$ ). Hence the potential energy is expressed as: $E_{p}=\int_{\zeta}^{0} g(\mathrm{~d} \bar{\rho} / \mathrm{d} z) z \mathrm{~d} z$. We shall assume that the background density profile is linear so that $\mathrm{d} \bar{\rho} / \mathrm{d} z$ is constant. Introducing the Brünt-Väisälä frequency $N^{2}=\left(-g / \rho_{0}\right) \mathrm{d} \bar{\rho} / \mathrm{d} z$, one gets $E_{p}=0.5 \rho_{0} N^{2} \zeta^{2}$. The generalized stiffness is therefore $0.5 \rho_{0} N^{2}$.

Let us now compute the kinetic energy to get the generalized inertia. The motion induced by a small amplitude monochromatic internal gravity wave occurs in a two-dimensional vertical plane, made by $\mathbf{k}$ and $\mathbf{g}$ (this is the propagation plane of the wave). We assume that this plane coincides with the $(x, z)$ plane. Hence, the kinetic energy is $E_{k}=0.5 \rho_{0}\left(u^{2}+w^{2}\right)$. From continuity equation: $k_{x} u+k_{z} w=0$, implying that $u=-\tan \theta w$, where $\theta$ is the angle of the wave vector with the horizontal plane. Since, $w=\dot{\zeta}$, one gets $E_{k}=0.5 \rho_{0} \dot{\zeta}^{2} / \cos ^{2} \theta$. The generalized inertia is therefore $0.5 \rho_{0} / \cos ^{2} \theta$.

It follows from (1) that the dispersion relation of a monochromatic internal gravity wave in a uniformly stratified medium (that is, with constant $N$ ) is

$$
\Omega^{2}=N^{2} \cos ^{2} \theta
$$

Relation (2) shows that the frequency depends upon the angle that the wave vector makes with the horizontal plane, which is a consequence of the anisotropy of the restoring force. There is no scale selection: when an oscillatory motion is imposed in a uniformly stratified fluid, any wave vector may a priori be selected provided its inclination satisfies the dispersion relation. The reason is that only a time scale is imposed, namely $N^{-1}$, but no length (or velocity) scale. In practice, a length scale is imposed by the geometry of the oscillating object ([11]) or by the size of the fluid container ([12]).

The laboratory experiment performed by Mowbray and Rarity [11] nicely illustrates the dispersion relation. A horizontal cylinder oscillates vertically at frequency $\Omega$ in a uniformly stratified fluid. For $\Omega<N$, internal gravity waves are generated, which carry the energy away from the source at the group velocity. Fig. 1(a) displays the fluctuating density field in a vertical plane perpendicular to the cylinder. The figure shows that the energy is localized in thin beams, whose angle with vertical is $\theta$. The velocity field induced by the waves is aligned with the beam direction and, as we discuss it in the next section, the beam width is eventually set by the diameter of the cylinder.

When internal gravity waves are generated in a rotating container, with angular frequency $\omega$, the Coriolis force acts as a second restoring force, which superimposes upon the buoyancy force. The dispersion relation becomes

$$
\Omega^{2}=N^{2} \cos ^{2} \theta+4 \omega^{2} \sin ^{2} \theta
$$

from which it can be inferred that

$$
4 \omega^{2} \leqslant \Omega^{2} \leqslant N^{2}
$$

As opposed to relation (2), relation (3) was only recently verified by laboratory experiments ([13]).

\subsection{Structure of the emitted wave field}

The two-dimensional laboratory experiment of Mowbray and Rarity [11] have also provided the basis for developing a theory describing the wave field emitted by an oscillating cylinder in a viscous stably-stratified fluid (with constant $N$ ), first in the far-field ([14]) and later in the near-field ([15]). In the far-field theory, the width of the beam is a self-similar function that involves the width of the viscous boundary layer $\delta$, the distance along the beam from the object $s$ and the angle of the wave beam. $\delta$ is defined as $\sqrt{v / \Omega}$, where $v$ is the kinematic viscosity of the fluid and $\Omega$ is the oscillating frequency of the cylinder. Hence, in the far-field theory, the beam width does not depend upon the scale of the object, which is therefore seen as a point source. In the near-field theory by contrast, as summarized by Gostiaux [16], the structure of the wave beam depends both upon the ratio $d / s$, where $d$ is the diameter of the cylinder and on the ratio $\delta / d$. The theory assumes that $\delta$ is much smaller than $d$. In the laboratory experiment of Mowbray and Rarity [11] where water is stably-stratified with salt, a typical value for $\Omega$ is $0.35 \mathrm{rad} / \mathrm{s}$ leading to $\delta=1.7 \mathrm{~mm}$, while $d=2 \mathrm{~cm}$. In the latter experiment, each beam (emitted on each side of the object and tangent to it) actually displays two maxima near the cylinder, which eventually merge into a single one away from the cylinder under the action of 


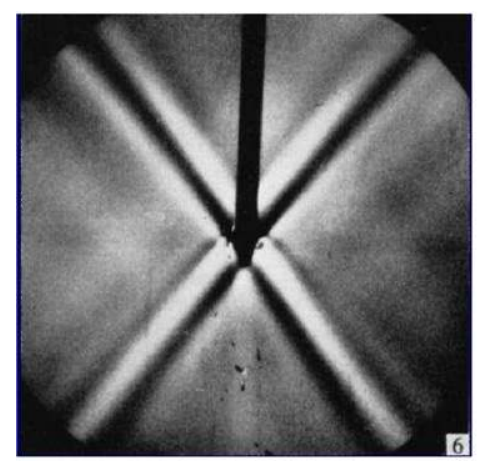

(a)

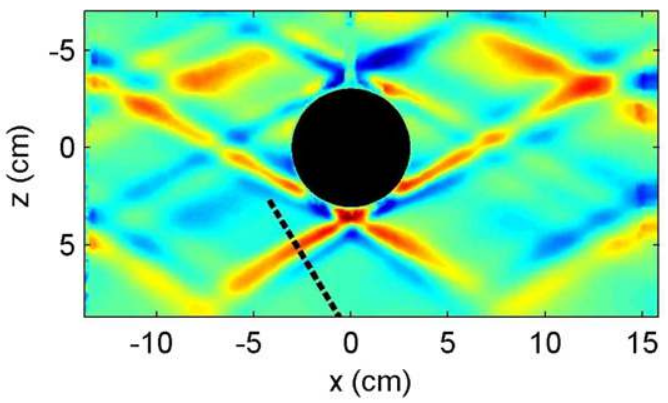

(b)

Fig. 1. Laboratory experiments of an oscillating horizontal cylinder (with axis perpendicular to the page) in a uniformly stratified fluid. (a) Cylinder of diameter $2 \mathrm{~cm}$ and oscillating frequency $0.35 \mathrm{rad} / \mathrm{s}$ (the dark vertical bar is the cylinder support); the black and white colors in the emission regions correspond to phases of opposite sign (from [11]). (b) Cylinder of diameter $6 \mathrm{~cm}$ and oscillating frequency $0.2 \mathrm{rad} / \mathrm{s}$; phases of opposite sign are marked by red and blue colors (from [16]).

Fig. 1. Emission d'ondes internes par un cylindre horizontal d'axe perpendiculaire à la figure oscillant verticalement dans un fluide uniformément stratifié. (a) Cylindre de diamètre $2 \mathrm{~cm}$ oscillant à la fréquence $0,35 \mathrm{rad} / \mathrm{s}$ (la ligne verticale sombre est la tige d'attache du cylindre); les couleurs blanche et noire dans les régions d'émission correspondent à des valeurs opposées de la phase (extrait de [11]). (b) Cylindre de diamètre $6 \mathrm{~cm}$ oscillant à la fréquence $0.2 \mathrm{rad} / \mathrm{s}$; les couleurs rouge et bleu correspondent à des valeurs opposées de la phase (extrait de [16]).

viscosity. For a very large cylinder, the two maxima are well pronounced, which result in two distinct wave beams on each side of the object. Hence, in the neighbourhood of the cylinder, eight beams can be observed (Fig. 1(b)).

The case of a three-dimensional oscillating object has been addressed by Voisin [17], for a sphere. Voisin computed the structure of the emitted wave field in a uniformly stratified fluid, taking into account unsteady and viscous effects. The case of an oscillating bounded object of arbitrary shape is under investigation ([18]). The results presented in Fig. 1(b) for a cylinder of large diameter suggest that the radius of curvature of the object at the location where the beam direction is tangent should come into play in the theory.

\section{Parametric instability of a monochromatic internal gravity wave}

\subsection{Numerical evidence for the parametric instability}

As mentioned in the Introduction, a monochromatic internal gravity wave of arbitrary amplitude may be unstable in a perfect fluid. More precisely, let us define the local wave steepness $s$ as the ratio of the wave-induced velocity along one direction divided by the phase velocity along that same direction. This definition implies that constant density surfaces are locally overturned for $s>1$, not overturned for $s<1$ and locally vertical for $s=1$. Using stability analysis, Mied [6] showed that internal gravity waves are unstable to two-dimensional perturbations for $s<1$ (that is, the instability develops in the propagation plane $(\mathbf{k}, \mathbf{g})$ of the wave). For $s>1$, Klostermeyer [7] proved that the waves are unstable to three-dimensional perturbations.

The resonant interaction (RI) theory, which predicts how energy may be transferred among a triad of waves of infinitely small steepness, provides a useful tool to analyse the instability of a wave. When two members of the triad are of much smaller steepness than the third one, referred to as the primary wave, the latter wave may be unstable to arbitrarily large wavenumbers of the two members, referred to as the secondary waves. The RI theory permits one to derive the expression of the growth rate of the secondary waves. This is illustrated in Fig. 2, where the growth rate is plotted versus the wavenumber of one secondary wave, both in a perfect fluid and taking into account molecular effects. When molecular effects are ignored, the growth rate saturates to a limit value as the wavenumber goes to infinity: any secondary wave of arbitrary large wavenumber may be excited by the instability. When viscosity is added, the largest wavenumbers are damped and a limited range of wavenumbers is actually excited. Viscosity therefore selects the most amplified wavenumbers. In a numerical simulation, the grid size provides an additional selection mechanism. The results displayed in Fig. 2 stem from a numerical simulation of an unstable monochromatic 


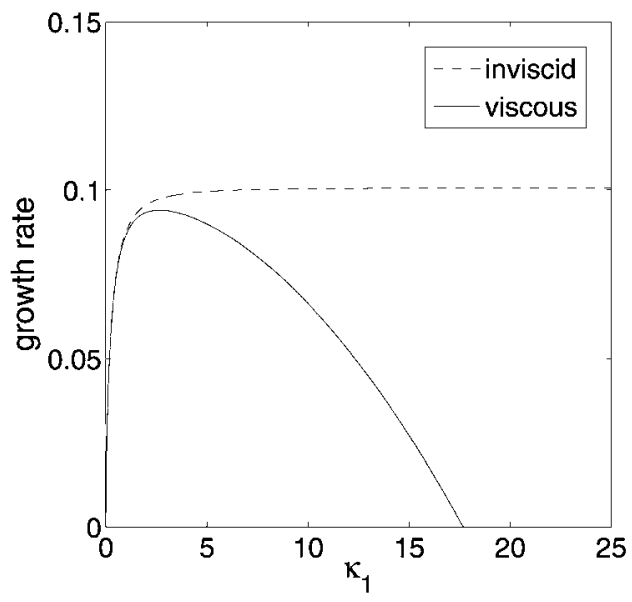

Fig. 2. Theoretical prediction (by resonant interaction theory) of the inviscid and viscous growth rates of a secondary wave amplified through parametric instability of a primary wave, as a function of the wavenumber $\left|\mathbf{k}_{1}\right|$ (denoted $\kappa_{1}$ ) of the secondary wave (from [19]).

Fig. 2. Prédiction théorique (par la théorie des interactions résonantes) des taux de croissance visqueux et non visqueux d'une onde secondaire amplifiée par l'instabilité paramétrique d'une onde interne de gravité ; les taux de croissance sont tracés en fonction du nombre d'onde de l'onde secondaire (extrait de [19]).

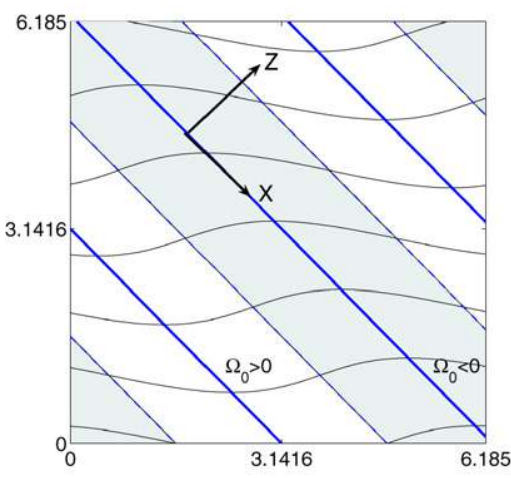

(a)

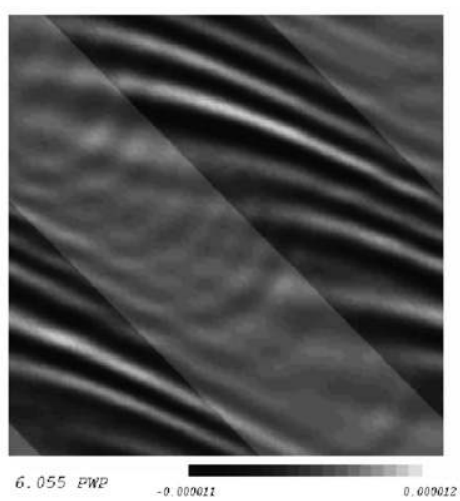

(b)

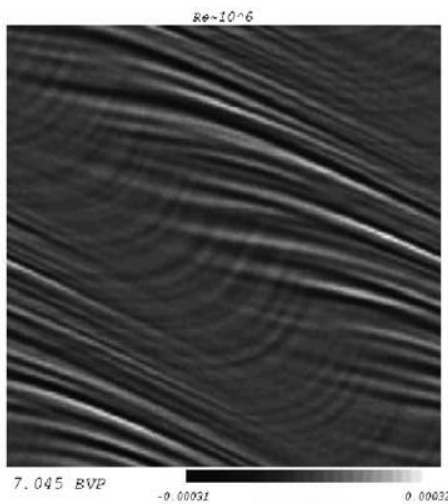

(c)

Fig. 3. (a) Structure of a primary wave with wave vector inclined at $45^{\circ}$ to the horizontal. Constant density contours and constant vorticity lines are shown. Regions of positive $\left(\Omega_{0}>0\right)$ and negative $\left(\Omega_{0}<0\right)$ primary wave vorticity are indicated by white and grey regions respectively. (b) Perturbation vorticity field at about 7 primary wave periods for run 1 ; regions of negative and positive primary wave vorticity are superimposed as dark and light bands respectively. (c) Same as (b) except that the value of the viscosity is smaller by two orders of magnitude (the primary wave vorticity is not superimposed) (from [19]).

Fig. 3. (a) Structure d'une onde primaire de vecteur d'onde incliné à $45^{\circ}$ par rapport à l'horizontal. Plusieurs lignes de densité constante sont visualisées. Les zones grisées et non grisées correspondent aux régions de vorticité négative et positive respectivement de l'onde primaire. (b) Champ de vorticité de la perturbation à un instant proche de 7 périodes de l'onde primaire pour le calcul 1. Les régions de vorticité négative et positive de l'onde primaire sont superposées et représentées par des bandes de couleur gris sombre et gris clair respectivement. (c) Idem (b) à l'exception de la valeur de la viscosité, qui a été diminuée d'un facteur 100 (la vorticité de l'onde primaire n'est plus superposée) (extrait de [19]).

$s_{0}=0.25$ internal gravity wave, with wave vector making an angle $\theta_{0}=45^{\circ}$ with the horizontal. This numerical simulation is referred to as run 1 in the following.

A few contours of the density field and of the vorticity field $\Omega_{0}$ of this primary wave are superimposed in Fig. 3(a). This wave is a solution of the nonlinear Boussinesq equations so that a perturbation had to be added at initial time to destabilize it. Fig. 3(b) displays the vorticity of the perturbation, as computed from run 1, after 7 periods of the primary wave: this vorticity is manifested as thin inclined bands whose angle with horizontal, $\theta_{1}$ say, satisfies $\cos \theta_{1}=\left(\cos \theta_{0}\right) / 2$; that is, using the dispersion relation, the perturbation frequency is half the primary wave vorticity. 
The thickness of the vorticity bands is selected by viscosity; indeed, Fig. 3(c) displays the same field except that the viscosity in the numerical simulation has been decreased by a factor 100: the same organization is obtained, with thinner bands. Also (not shown), the vorticity bands change sign after one primary wave period. All these observations are consistent with the perturbation growing from parametric instability, the vorticity band width being half the wavelength selected by viscosity.

The numerical simulations performed by Koudella and Staquet [19] show that the perturbation growth is controlled by a few parametrically resonant triads of waves, which may be assumed to be independent as they grow. The secondary waves of these triads have very close wavenumbers (because of viscosity selection) and therefore very close growth rates. This accounts for the very deterministic structure of the perturbation displayed in Fig. 3(b) (and, to a lesser extent, in Fig. 3(c)).

Fig. 3(b) also shows that the perturbation vorticity is maximum at locations where the primary wave vorticity is negative. This remark is actually the key for optimum parametric amplification, as we show it now.

\subsection{Mechanisms of the parametric instability}

In this section, we analyse the mechanism through which the perturbation grows. As usual in this type of study, we consider the energetics of the perturbation. We start with a rotation of the reference frame (see [6]) so that $Z$ is aligned with the primary wave vector in the new $(0, X, Z)$ frame (see Fig. 3(a)). We decompose the velocity and density fields into a part associated with the primary wave, denoted $\left[\left(U_{0}, 0\right), R_{0}\right]$, and a part associated with the perturbation, denoted $\left[\left(U^{\prime}, W^{\prime}\right), \rho^{\prime}\right]$. The Boussinesq equations linearized about the primary wave state and written for the kinetic and potential energy of the perturbation immediately yield that (i) the kinetic energy of the perturbation is forced by the term $-U^{\prime} W^{\prime} \mathrm{d} U_{0} / \mathrm{d} Z$, which involves the shear of the primary wave $\mathrm{d} U_{0} / \mathrm{d} Z\left(=\Omega_{0}\right)$ as for any unstable shear flow and (ii) the potential energy is forced by the term $N^{-2} \rho^{\prime} W^{\prime} \mathrm{d} R_{0} / \mathrm{d} Z$, which involves the vertical density gradient of the primary wave $\mathrm{d} R_{0} / \mathrm{d} Z$ ([20]).

Let us consider the forcing of the kinetic energy. Since the perturbation consists of secondary internal gravity waves, the correlation $U^{\prime} W^{\prime}$ is one-signed. It is not difficult to show here that $U^{\prime} W^{\prime} \geqslant 0$ : the velocity field of the perturbation is aligned with the vorticity bands displayed in Fig. 3(b) so that $U^{\prime}$ has the same sign as $W^{\prime}$. However, $\mathrm{d} U_{0} / \mathrm{d} Z$ oscillates in time and space. At a position where $\mathrm{d} U_{0} / \mathrm{d} Z$ is negative, the forcing term $-U^{\prime} W^{\prime} \mathrm{d} U_{0} / \mathrm{d} Z$ is positive, i.e. the perturbation is forced; but the forcing term becomes negative when $\mathrm{d} U_{0} / \mathrm{d} Z$ becomes positive at that position (half a primary wave period later) implying that the perturbation should give back the acquired energy. One may wonder, then, how a nonzero net forcing is possible.

The point is that the perturbation will not give its kinetic energy back if the correlation term $U^{\prime} W^{\prime}$ is zero, or very small, during the half primary wave period where $\mathrm{d} U_{0} / \mathrm{d} Z$ is positive. This will be the case if the perturbation energy is of potential form during this half-period. It follows that optimum forcing occurs when the perturbation energy is of kinetic form during the half-period when $\mathrm{d} U_{0} / \mathrm{d} Z<0$ and of potential form during the other half-period when $\mathrm{d} U_{0} / \mathrm{d} Z>0$.

Since $\mathrm{d} U_{0} / \mathrm{d} Z$ is of the form $\exp \left(\mathrm{i} \Phi_{0}\right)$, where $\Phi_{0}$ is the phase of the primary wave, the energy of the perturbation should be of this form too. It follows that the velocity and density fields of the perturbation should behave as $\exp \left(\mathrm{i} \Phi_{0} / 2\right)$. In other words, the instability must be of the parametric type for optimal forcing to occur.

What about the potential energy of the perturbation? It can be shown that its forcing term $N^{-2} \rho^{\prime} W^{\prime} \mathrm{d} R_{0} / \mathrm{d} Z$ is always positive. Since $\mathrm{d} R_{0} / \mathrm{d} Z$ oscillates at the primary wave period, this implies that the perturbation potential energy is forced twice per primary wave period: at the locations where $\mathrm{d} R_{0} / \mathrm{d} Z$ is maximum, where the vertical density gradient experienced by the perturbation is decreased, and at locations where $\mathrm{d} R_{0} / \mathrm{d} Z$ is minimum, where the vertical density gradient experienced by the perturbation is increased. The former region is referred to as RSS (reduced static stability regions) and the latter as ISS (increased static stability regions). By contrast, as shown above, the kinetic energy of the perturbation is forced once, in regions where the primary wave shear $\mathrm{d} U_{0} / \mathrm{d} Z$ is negative, and this is where vorticity bands are visible in Fig. 3(b). Due to the conversion of kinetic energy into potential energy, it can be shown that the potential energy of the perturbation is converted into kinetic energy in a ISS region, the reverse occurring in a RSS region. As a consequence, the perturbation potential energy always increases in RSS regions.

A summary of the spatial occurrence of these forcing mechanisms is displayed in Fig. 4. 


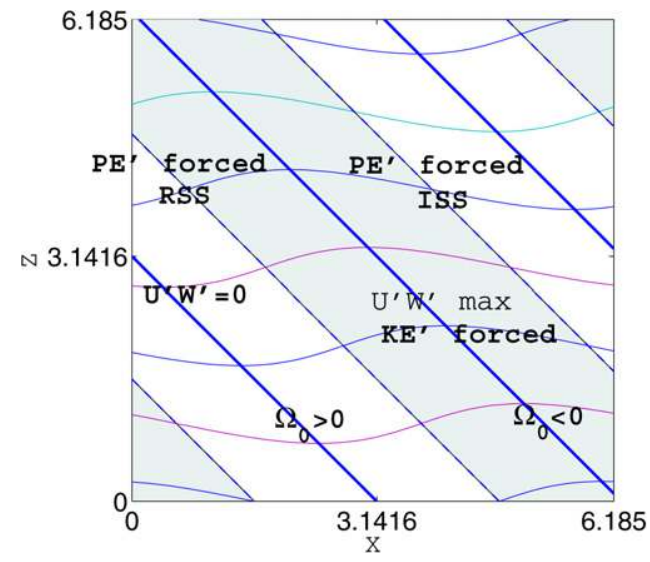

Fig. 4. Same as Fig. 3(a), with regions where either the kinetic energy or the potential energy of the perturbation is forced by parametric instability. At a given time, kinetic energy is forced in regions where the primary wave vorticity $\Omega_{0}$ is negative; potential energy is forced in regions where the vertical density gradient of the primary wave is either maximum (reduced static stability region or RSS) or minimum (increased static stability region or ISS).

Fig. 4. Idem Fig. 3(a), avec l'ajout des régions dans lesquelles l'énergie cinétique ou l'énergie potentielle de la perturbation est forcée par l'instabilité paramétrique. A un instant donné, l'energie cinétique est forcée dans les régions où la vorticité de l'onde primaire $\Omega_{0}$ est négative; l'énergie potentielle est forcée dans les régions où le gradient vertical du champ de densité de l'onde primaire est maximal (région de stabilité statique réduite—RSS) ou minimal (région de stabilité statique augmentée-ISS).

\subsection{Kinematic modelling of the parametric instability}

In the following, a parametrically resonant triad $\left[\left(\mathbf{k}_{0}, \Omega_{0}\right),\left(\mathbf{k}_{1}, \Omega_{1}\right),\left(\mathbf{k}_{2}, \Omega_{2}\right)\right]$ refers to three waves consisting of one primary wave $\left(\mathbf{k}_{0}, \Omega_{0}\right)$ and two secondary waves forced by the primary wave through parametric instability. Since gravity waves are considered, $\Omega_{i}$ is related to $\mathbf{k}_{i}, 0 \leqslant i \leqslant 2$, by the dispersion relation (2). Because a wave triad is considered, one has $\mathbf{k}_{0}+\mathbf{k}_{1}+\mathbf{k}_{2}=\mathbf{0}$. Finally, since the instability is of the parametric type, one has $\left|\Omega_{1}\right| \simeq\left|\Omega_{2}\right| \simeq$ $\left|\Omega_{0} / 2\right|$.

As we recalled it above, the perturbation growth is controlled by a few parametrically resonant wave triads. It follows that the energetics of the perturbation may be reduced to that of a single resonant triad. Approximate expressions of the perturbation energy and of energy transfer terms between the primary wave and the perturbation may be derived from this assumption, as we discuss now.

Because small scale waves are most amplified by the instability, we shall assume that $\left|\mathbf{k}_{1}\right|,\left|\mathbf{k}_{2}\right| \ll\left|\mathbf{k}_{0}\right|$ with $\mathbf{k}_{1} \simeq$ $-\mathbf{k}_{2}$. From these assumptions and the properties of a parametrically resonant triad, it can be shown that the phase average over $2 \pi$ of the ratio of the kinetic energy transfer term to the potential energy transfer term is smaller than 1 . More precisely

$$
\frac{\left\langle-U^{\prime} W^{\prime} \mathrm{d} U_{0} / \mathrm{d} Z\right\rangle}{N^{-2}\left\langle\rho^{\prime} W^{\prime} \mathrm{d} R_{0} / \mathrm{d} Z\right\rangle}=\cos \left(\theta_{1}-\theta_{0}\right),
$$

where \langle\rangle denotes the phase average over $2 \pi$. For the $45^{\circ}$ primary wave vector considered above, this ratio has a value of 0.91 . We therefore conclude that for an unstable primary wave of small amplitude, potential energy is transferred toward small scales at a higher rate than kinetic energy. This is actually a very general result in stratified turbulence, where it is found that potential energy transfers toward small scales are higher than kinetic energy transfers. Two arguments were provided to account for this behaviour: the incompressibility constraint, which applies to the velocity but not to the density field ([21]) and the subset of two-dimensional interactions which prevent kinetic energy from being transferred toward small scales ([22]).

What does happen when the perturbation amplifies? Its vorticity field in $\mathrm{d} U_{0} / \mathrm{d} z<0$ regions may become unstable to a (large scale) Kelvin-Helmholtz instability. On the other hand, the perturbation potential energy continuously increases in RSS regions, eventually leading to statically unstable fluid layers (that is, heavy fluid lies over light fluid); hence, a (small-scale) buoyancy-induced instability may also occur there. In a three-dimensional fluid, both instabilities therefore compete. Koudella and Staquet [19] showed that the buoyancy-induced instability grows faster 
than the Kelvin-Helmholtz instability, unless viscous effects are strong enough, thereby confirming earlier heuristic analysis ([23]). It follows that an unstable primary wave of small steepness eventually breaks down through a three-dimensional buoyancy-induced instability (if viscous effects are low enough). It should be noted that the buoyancy-induced instability develops in a plane perpendicular to the primary wave propagation plane so that, if the simulation of the flow dynamics is limited to this propagation plane (two-dimensional computation), the KelvinHelmholtz instability is selected by the two-dimensional geometry. In other words, the primary wave breaks through Kelvin-Helmholtz instability in this case.

\section{Turbulence and mixing induced by internal gravity waves}

Breaking internal gravity waves lead to turbulence and we saw in the previous section, using a deterministic point of view, how a monochromatic internal gravity wave may break. The turbulence resulting from breaking gravity waves has peculiar properties, which can be accounted for by noting that breaking occurs when nonlinear effects become of the same order as stratification effects. Namely, the advective time scale $L / U$, where $L=1 / k$ is the typical scale of the wave and $U$ is the typical wave-induced velocity, is of the order of $N^{-1}$ :

$$
k U \simeq N
$$

In a statistical description of the wave field, the energy density spectrum $E(k)$ is introduced, with the velocity $U$ at scale $1 / k$ being linked to the energy density spectrum at that scale by $U(k)=\sqrt{k E(k)}$ (see f.i. [21]). Relation (6) then yields

$$
E(k) \simeq N^{2} k^{-3}
$$

which is the spectrum characterizing 'internal gravity wave turbulence'. It is remarkable that not only is this spectral law found in natural media and experiments, but the level of the spectrum is also fixed, being close to 0.2. Namely, both the kinetic and potential energy spectra scale like $0.2 N^{2} k^{-3}$. Since vertical scales are much smaller than horizontal scales in a stratified flow, the wavenumber is actually dominated by its vertical component so that these spectrum are usually expressed as a function of the vertical component of the wave vector $k_{z}$. The observation of this spectrum is illustrated in Fig. 5, which displays the potential energy spectrum in the ocean (frame (a)), in the stratosphere (b) and in a laboratory (c) and numerical experiment (d). In frames (a), (b) and (d), the horizontal axis is the vertical component of the wave vector. In (c), the spectra are plotted instead as a function of the forcing frequency $f$ of the waves and display a $f^{-3}$ spectrum for different values of the parameters of the experiment; a $k_{z}^{-3}$ spectrum is simply obtained via a Taylor assumption.

The derivation of the spectral law (7), and corrections to it, have been the subject of an abundant literature in the atmospheric community. The derivation we proposed above is rather crude, but easy to follow, and several models have been proposed to get this law. We refer to the paper by Fritts and Alexander [24] for more detail.

It should be pointed out that some authors do not consider the spectral law (7) to characterize internal gravity wave turbulence. Indeed, in some cases of forced stratified turbulence, a different spectral dependency is found despite the flow is designed to mostly consist of breaking internal gravity waves (see [25]).

In general, the internal gravity wave field does not account for all of the fluctuations with respect to the hydrostatic equilibrium state. Another component of motion exists, which is complementary to the wave field in the sense that is does not propagate. This motion, referred to as the 'vortex' or 'vortical' part of the flow, may be characterized by its containing all of the potential vorticity of the flow, defined as $((\nabla \wedge \mathbf{u}) . \nabla \rho) / \rho_{0}$ in the Boussinesq approximation. Besides the wave and vortex motions, a mean horizontal flow often exists. The vortex mode is a slow motion, with typical time scale much larger than $N^{-1}$ and with velocity field lying along the isopycnals when the fluid is strongly stratified (see [26]). We shall not discuss this topic in the present paper and refer to the review article by Lelong and Riley [27] and to the paper by Cambon [28].

Mixing in a stably-stratified fluid results from a diffusive flux of mass, which occurs across the isopycnals. Internal gravity waves can break and mix the fluid whatever the stratification level. This accounts for the strong interest of the oceanographic community in the role of internal gravity waves in oceanic mixing, as we discuss in the next section. Up to now, mixing by internal gravity waves has been rarely addressed (see [29] for a review). Internal gravity waves break through a dynamical instability, which is a buoyancy-induced instability (heavy fluid lies over light fluid). One may wonder whether other dynamical instabilities may result in mixing in a rotating stratified fluid (without mean 


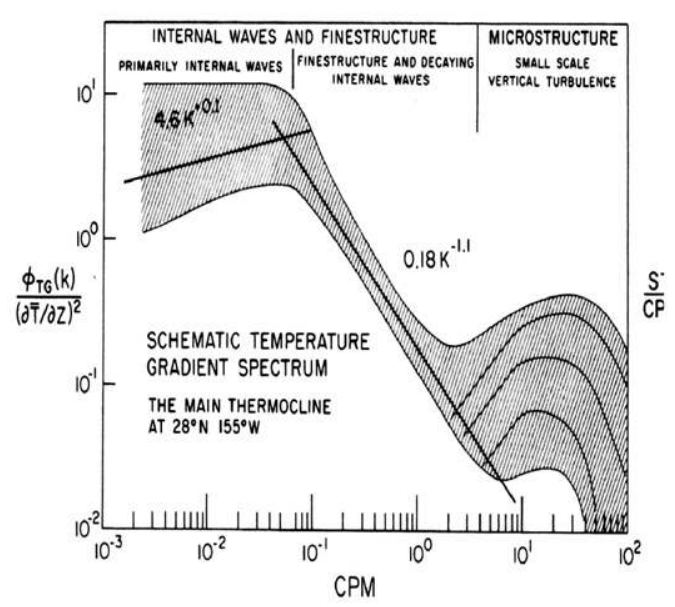

(a)

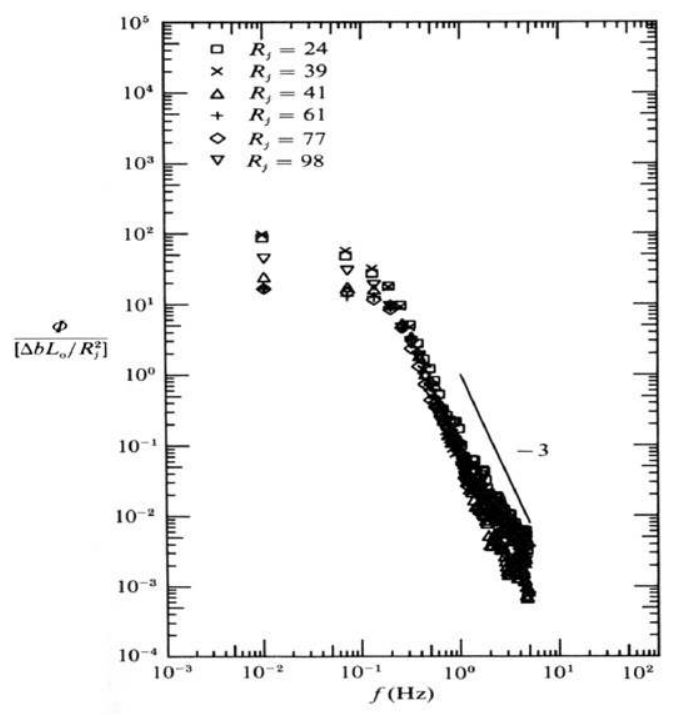

(c)

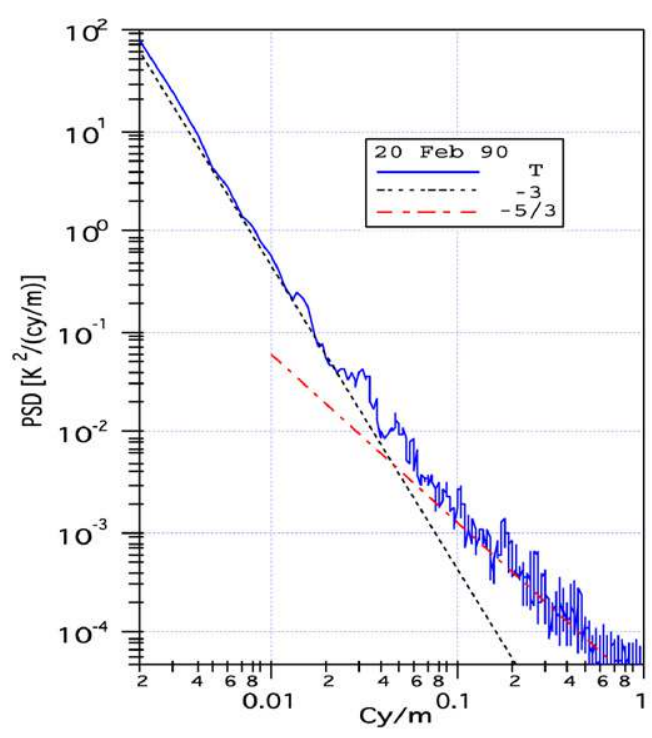

(b)

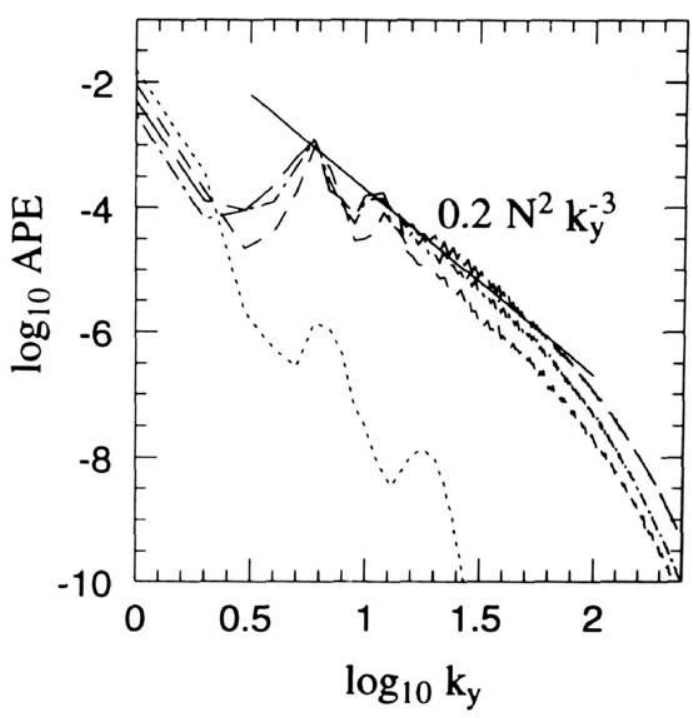

(d)

Fig. 5. Evidence of $k_{z}^{-3}$ potential energy spectra in the presence of internal gravity wave breaking. (a) Schematic spectra of vertical temperature gradients $k_{z}^{2} E_{p}\left(k_{z}\right)$ in the oceanic thermocline (from [33]); in this frame and in (b), the vertical wavenumber is expressed in cycle/m (cpm). (b) Stratospheric temperature spectra measured from instrumented balloons, showing a $k_{z}^{-3}$ range with a Kolmogoroff $k_{z}^{-5 / 3}$ tail at high $k_{z}$ (provided by F. Dalaudier). (c) Normalized internal gravity wave spectra, computed from fluoresceine concentration profiles in a laboratory experiment of mixing of a density interface by an oscillating grid. $\Delta b$ is the buoyancy jump across the density interface, $l_{0}$ is an integral scale of turbulence and $R_{j}$ is a Richardson number (from [34]). Note that frequency spectra are plotted. (d) Two-dimensional direct numerical simulations of a large scale breaking internal gravity wave ( $k_{y}$ is the vertical wavenumber and APE refers to the available potential energy) (from [35]).

Fig. 5. Mesures de spectres d'énergie potentielle en $k_{z}^{-3}$ lors du déferlement d'ondes de gravité internes. (a) Spectre des gradients verticaux de température $k_{z}^{2} E_{p}\left(k_{z}\right)$ mesurés dans la thermocline océanique (extrait de [33]); dans cette figure et la suivante, le nombre d'onde vertical est exprimé en cycle $/ \mathrm{m}$ (cpm). (b) Spectre de température mesuré dans la stratosphère à partir de ballons instrumentés ; le spectre en $k_{z}^{-3}$ se termine par un spectre de Kolmogoroff $k_{z}^{-5 / 3}$ aux petites échelles (figure fourni par F. Dalaudier). (c) Expérience de laboratoire du mélange d'une interface en densité par une grille oscillante, dans un régime où les mouvements à l'interface sont des ondes internes non linéaires. Les spectres, fonction ici de la fréquence, sont obtenus à partir de profils de concentration de fluorescéine (extrait de [34]). (d) Simulations numériques directes bidimensionnelles du déferlement d'une onde de gravité interne; $k_{y}$ est le nombre d'onde vertical et APE dénote l'énergie potentielle disponible (extrait de [35]). 
shear). In a nonrotating stratified fluid, a Kelvin-Helmholtz instability associated with the vertical shear of the vortex mode has been put to the fore ([30]) and shown to contribute to most of the kinetic energy dissipation ([31]). When rotation is added, the Coriolis force does not modify the energy content of the fluid system, since it does not work, but it does modify the energy transfers. The Kelvin-Helmholtz instability just mentioned might then be suppressed. Indeed, as shown experimentally by Praud et al. [32], when rotation is added in a turbulent stably-stratified fluid, energy transfers toward small scales are inhibited (relative to the nonrotating case). It follows that fluid mixing, which occurs at the dissipative scales, should be reduced as well. Hence, in a rotating stably-stratified fluid, internal gravity wave breaking could be the only major process for fluid mixing.

\section{Application to the ocean: the internal tide and deep ocean mixing}

\subsection{The internal tide}

The interaction of the tide with bottom topography in the ocean creates internal gravity waves. One way to understand this generation process is to consider a frame of reference attached to the tide. In this frame, the topography, say a submarine mountain or a continental shelf, behaves as a horizontally oscillating body in a stratified fluid. This change of reference frame is not Galilean since the body is accelerated but we shall assume this acceleration may be neglected and the reference frame be considered as Galilean. We can therefore rely on the laboratory experiments described in Section 2.1 to predict the internal wave behaviour: rays should be emitted from locations on the object where the tangent to the object coincides with the direction predicted by the dispersion relation. Fig. 6(a) illustrates this situation for a continental shelf, when the linear Boussinesq equations are solved. (Note that the shelf slope is a few degrees only as it extends over $50 \mathrm{~km}$ from a maximum depth of $4000 \mathrm{~m}$ up to $200 \mathrm{~m}$.) Only one beam is generated since there is only one point of tangency of the beam with the topography. The remarkable feature of this figure is that the beam displays a well-defined width. In this linear description, the beam transfers energy away from its generation region by reflecting on the upper and lower boundaries of the ocean.

Nonlinear effects modify the structure of the beam. However, a wave beam in an inviscid, uniformly stratified medium is an exact solution of the Boussinesq equations if the medium is infinite ([36]). Boundaries may therefore lead to nonlinear effects and this is indeed what is observed. Thus (not shown), a beam at twice the tidal frequency may be produced at the generation region. At the bottom boundary, the nonlinear interaction of the incident beam with the

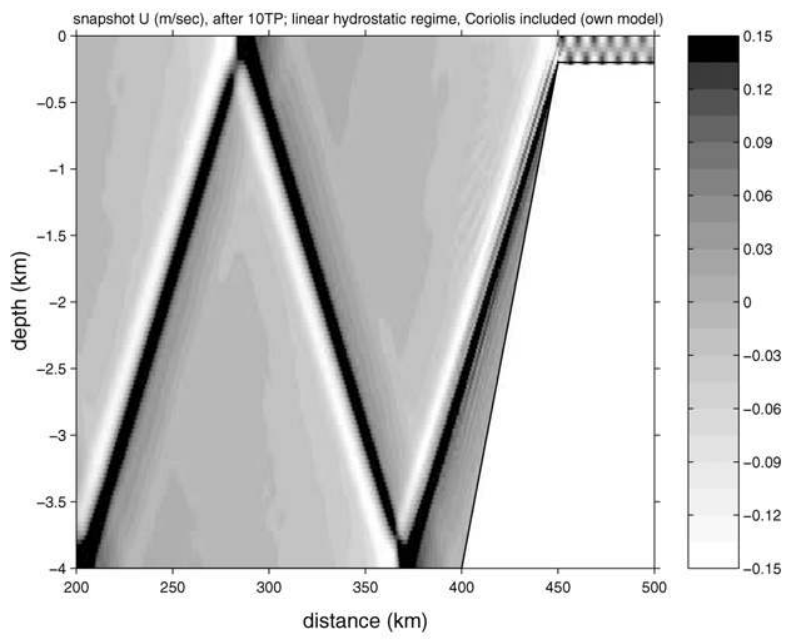

(a)

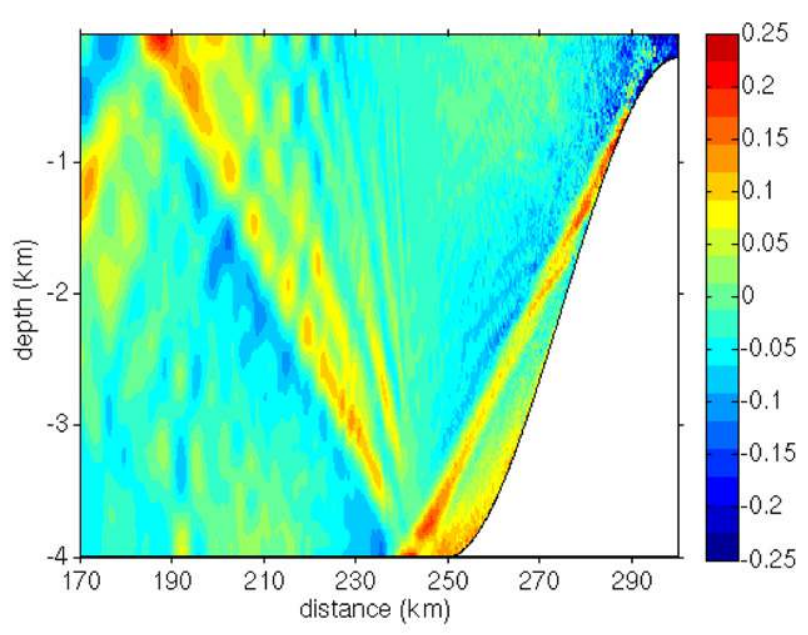

(b)

Fig. 6. (a) Wave-field emitted from a continental shelf, in the linear hydrostatic regime (simulation performed by Theo Gerkema). (b) Same as (a) in the nonlinear non-hydrostatic regime. The horizontal component of the velocity field is plotted after 10 tidal periods.

Fig. 6. (a) Champ d'ondes de gravité internes émis à partir d'un talus continental, en régime linéaire et hydrostatique (calcul réalisé par Theo Gerkema). (b) Idem (a) mais en régime non linéaire et non hydrostatique. Le champ de vitesse horizontale est tracé au bout de 10 périodes de forçage. 
reflected beam generates harmonics, with frequencies equal to $n \Omega_{\mathrm{M} 2}$, where $\Omega_{\mathrm{M} 2}$ is the tidal frequency (Fig. 6(b)). The number of harmonics depends upon the ratio $N / \Omega_{\mathrm{M} 2}$, since it cannot exceed this ratio. The values for $\Omega_{\mathrm{M} 2}$ and $N$ in the computation displayed in Fig. 6(b) are $1.4 \times 10^{-4}$ and $2 \times 10^{-3}$ respectively so that, with these values, up to 14 harmonics should be detected. The amplitude of the harmonics however decreases as the harmonics number increases and, in practice, the largest harmonics which could be detected is $10 \Omega_{\mathrm{M} 2}$.

\subsection{Parametric instability of the internal tide}

As already mentioned, the internal tide beam is an exact solution of the inviscid Boussinesq equations in an infinite medium, as is a monochromatic internal gravity wave field. Therefore, the emitted wave beam may be subjected to parametric instability. The upper cut-off frequency $N$ does not come into play in this process since the parametric instability excites waves with half the tidal frequency. Away from the equator, the Earth rotation influences the internal tide dynamics implying that, from relation (4), the Coriolis frequency $f$ acts as a lower cut-off frequency. Indeed, $\Omega_{\mathrm{M} 2} / 2$ should be larger than $f$ for the instability to be able to develop. With $\Omega_{\mathrm{M} 2}=1.4 \times 10^{-4}$, this implies that the instability can only develop at latitudes lower than $28.9^{\circ}$. This is the so-called 'critical latitude' and the corresponding value of the Coriolis frequency, defined as $f_{c}=\Omega_{\mathrm{M} 2} / 2$, is referred to as the critical frequency.

Let us estimate the group velocity of the parametrically amplified secondary waves. Since their frequency is low (close to $f$ ), their group velocity is nearly horizontal and a good estimate of this group velocity is provided by its horizontal component. This component is plotted in Fig. 7 versus the Coriolis frequency $f$ scaled by $f_{c}$ : it is maximum at the equator $(f=0)$ and vanishes at the critical frequency. This implies that, just below the critical latitude, a perturbation is parametrically amplified without propagation; the amplified energy therefore accumulates at the amplification location. By contrast, at the equator, the amplified perturbation propagates at the group velocity away from the generation region, making its detection more difficult.

These remarks are illustrated in Fig. 8, which displays results from two computations analogous to those displayed in Fig. 6: the internal tide is produced by the interaction of the barotropic tide (modelled by a barotropic forcing imposed at the 'open-ocean' vertical boundary) with a continental shelf. Constant contours of the horizontal velocity field filtered at frequency $\Omega_{\mathrm{M} 2} / 2$ are displayed in Fig. 8 at two different latitudes: just below the critical latitude (frame (a)) and at the equator (frame (b)). The filtering at frequency $\Omega_{\mathrm{M} 2} / 2$ amounts to selecting the parametrically amplified perturbation. When Fig. 8(a) is inspected, the flow structure already observed in Fig. 3(b) is recognized: the parametric instability is manifested as nearly-horizontal bands of alternate sign, which form within the envelope of the tidal beam, whose thickness should be imposed by the (turbulent, in the present case) viscosity. The amplified perturbation is less visible in frame (b), at the equator, because the perturbation slowly 'escapes' (at the group velocity) from the generation region. Note that, in the latter case, the M2 component of the velocity field is still visible very likely because the temporal average was performed over a too small number of tidal periods.

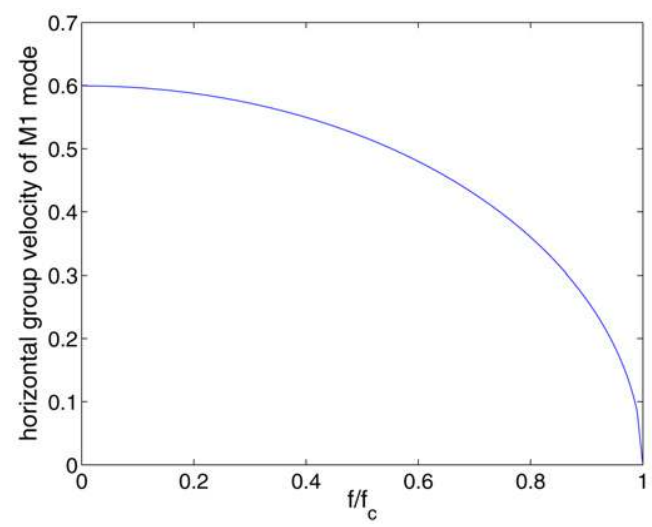

Fig. 7. Horizontal component of the group velocity of a parametrically amplified wave from a M2 wave beam, versus the Coriolis parameter $f$ normalized by $f_{c}=\Omega_{\mathrm{M} 2} / 2$. The parametrically amplified wave is referred to as the M1 mode.

Fig. 7. Composante horizontale de la vitesse de groupe d'une onde de gravité interne (dénommée « mode M1 ») amplifiée par instabilité paramétrique d'une onde de marée interne de fréquence $\Omega_{\mathrm{M} 2}$. L'axe horizontal représente le paramètre de Coriolis $f$ normalisé par $f_{c}=\Omega_{\mathrm{M} 2} / 2$. 


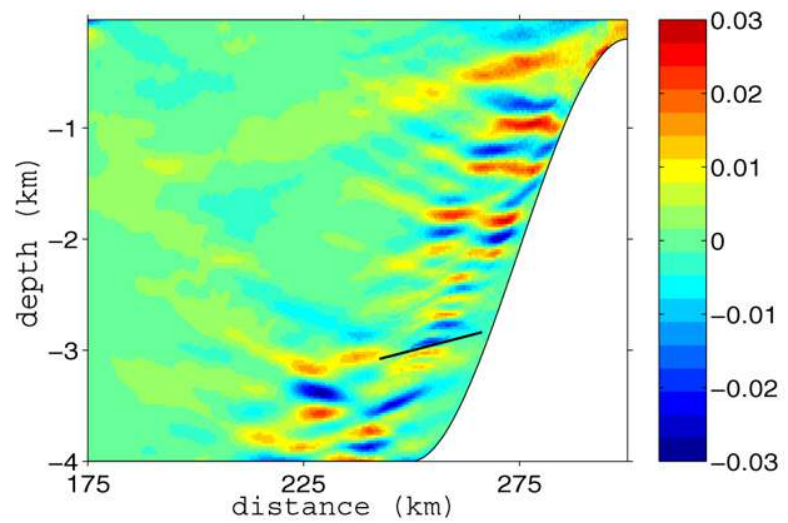

(a)

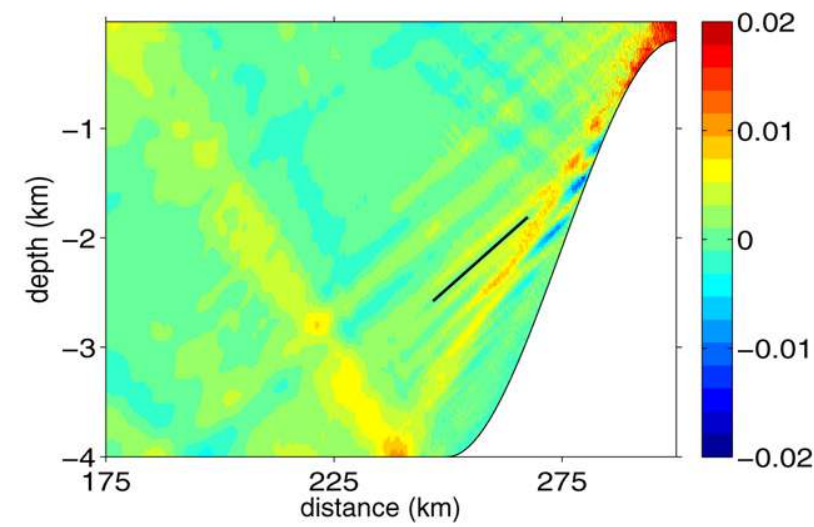

(b)

Fig. 8. (a) Horizontal velocity field filtered at frequency $\Omega_{\mathrm{M} 2} / 2$, for a computation with $f$ just below $f_{c}$; from a practical point of view, the horizontal velocity is multiplied by $\cos \left(0.5 \Omega_{\mathrm{M} 2} t\right)$ and averaged over tidal periods 7 to 23 . (b) Same as (a) for $f=0$; the temporal average is performed from 5 to 15 tidal periods. In both frames, the phase line direction predicted by the linear theory is indicated with a straight black line.

Fig. 8. (a) Composante horizontale du champ de vitesse filtré à la fréquence $\Omega_{\mathrm{M} 2} / 2$, pour une valeur de $f$ juste sous la fréquence critique $f_{c}$. (b) Idem (a) pour $f=0$.

\subsection{Mixing in the deep ocean}

A fascinating aspect of mixing in the deep ocean is its direct implication in the thermohaline circulation.

The thermohaline circulation designates a slow oceanic circulation (the typical time scale is one thousand years) at planetary scale, which is due to the sinking of cold dense water masses at high latitudes in each winter hemisphere. These cold water masses sink down to the oceanic bottom and travel equatorwards while slowly moving upwards toward the surface, through mixing. Mixing is an essential process in the thermohaline circulation: without turbulent mixing, the ocean would be a cold salty pool at rest, with a very small mixed upper layer due to wind and solar heating ([37]). The mean vertical temperature profile in the ocean is inconsistent with this view, implying that processes responsible for mixing account for the upwelling of the thermohaline circulation.

Knowledge in the ocean circulation partly stems from large scale numerical models. The best models today have a resolution of $1 / 10^{\circ}$, that is (since $1^{\circ}$ is about $100 \mathrm{~km}$ at the equator), a horizontal resolution of a few kilometers. Mixing processes need therefore to be parameterized in these models and the scientific community is currently engaged in an effort to identify mixing processes along with their impact on the ambient flow.

According to Wunsch and Ferrari [9], much, if not all the mixing energy is provided by the internal wave field. As we mentioned in Section 2.1, the two main sources of internal gravity waves in the deep ocean are the wind and the tide. Global estimates from satellite measurements point toward a possible balanced contribution of the two sources. Regarding the tidal contribution, the question is to quantify the role of internal tides into the abyssal mixing processes. A few questions need to be solved for this purpose: which part of (barotropic) tidal energy is transferred to internal tide? Which amount of internal tide energy contributes to mixing? How and where does mixing occur? These questions are currently addressed in the literature for two simple configurations, a submarine mountain ([38,39]) and a continental shelf $([40,41])$.

The interaction of the internal tide with topography induces mixing. When the generation region is considered, very small scales are produced (whose origin is not clear yet) as well as mixing ([40]). Another location where mixing is observed is where the incident beam interacts with the reflected beam. As discussed in Section 5.1, the superposition of these two beams leads to nonlinearity and higher wavenumbers (i.e. small scales) are produced, as well as higher frequencies. The generation of smaller scales occurs locally and dissipates the incident beam energy locally. By contrast, the generation of higher harmonics is associated with wave beams which carry the incident beam energy away. The analysis of Gerkema et al. [40] shows that about half of the energy lost by the incident beam upon reflexion is due to dissipation and half goes into higher harmonics.

The internal tide beam may transfer its energy through other processes: the parametric instability is one of them and has been illustrated above. The latter work confirms the conjecture by Hibiya et al. [42] that the intense mixing regions 
observed just below the critical latitude in the ocean, as inferred from a general circulation numerical model and from in situ measurements ([43]), may result from parametric instability of the internal tide. When a steep thermocline is present, like a seasonal thermocline, the interaction of the wave beam with the thermocline may generate solitons, which transport energy hundreds of kilometers away from their generation region. This mechanism has been predicted theoretically ([44]) but has not been verified through numerical or laboratory experiments. Yet there is a need to account for the generation of solitons in the Bay of Biscay for instance, away from any topography ([45]), and this mechanism would account for this observation. It is noteworthy that the theory of solitons relies on the KortewegDe Vries equation, which was first developed by Boussinesq (see [46] for a review).

\section{Conclusion}

The purpose of this article was to illustrate the very rich dynamics of internal gravity waves, which are solutions of the Navier-Stokes equations in the Boussinesq approximation. The very study of those waves started in the midsixties, when Ball [47] realized that two surface waves could resonantly interact with an internal gravity wave. Much has been learned about these waves, especially from a theoretical point of view, but the role of rotation on those waves remains to be explored. For instance, the dispersion relation of internal gravity waves subjected to rotation has been verified experimentally only recently ([13]). And much remains to be done when natural media are considered. In this paper, we discussed internal gravity waves in the deep ocean, where their fundamental role in mixing the abyssal water masses has been conjectured but not quantified. The sources of internal gravity waves in the atmosphere, other than the wind blowing over orography, are also the subject of active research. For instance, convective clouds at low latitudes generate such waves, which may interact in turn with the clouds. Also, the behaviour of these waves in the dynamics of the radiative zone of the Sun, which is a stably-stratified region, is totally unknown. From a modelling point of view, weakly nonlinear theories have been developed to account for the statistical properties of those waves (see [8]). However, from a practical point of view, no reliable parameterization of the waves exists, which would relate the transport coefficients they induce to the dynamical parameters of the large scale motions which create them. Such parameterizations are crucially needed in general circulation models of the ocean, as well as in the atmosphere.

\section{Acknowledgements}

The author thanks Bruno Voisin for useful discussions.

\section{References}

[1] M.J. Lighthill, Waves in Fluids, Cambridge University Press, 1978.

[2] A.E. Gill, Atmosphere-Ocean Dynamics, Academic Press, 1987.

[3] E. Danioux, P. Klein, P. Rivière, Propagation of wind energy into the deep ocean through mesoscale eddies, J. Phys. Oceanogr., submitted for publication.

[4] O.M. Phillips, Wave interactions - the evolution of an idea, J. Fluid Mech. 106 (1981) 215-227.

[5] P.G. Drazin, On the parametric instability of an internal gravity wave, Proc. R. Soc. Lond. A 356 (1977) 411.

[6] R.P. Mied, The occurrence of parametric instabilities in finite-amplitude internal gravity waves, J. Fluid Mech. 78 (1976) $763-784$.

[7] J. Klostermeyer, Two and three-dimensional parametric instabilities in finite amplitude internal gravity waves, Geophys. Astrophys. Fluid Dynam. 61 (2006) 1.

[8] C. Staquet, J. Sommeria, Mixing by breaking internal gravity waves: from instabilities to turbulence, Annu. Rev. Fluid Mech. 34 (2002) 559-593.

[9] C. Wunsch, R. Ferrari, Vertical mixing, energy and the general circulation of the oceans, Annu. Rev. Fluid Mech. 36 (2004) $281-314$.

[10] C. Staquet, Internal gravity waves in geophysical fluids, in: CISM Lecture Notes, vol. 479, Springer-Verlag, 2005 , pp. 75-131.

[11] D.E. Mowbray, B.S.H. Rarity, A theoretical and experimental investigation of the phase configuration of internal waves of small amplitude in a density stratified liquid, J. Fluid Mech. 28 (1967) 1-16.

[12] D. Benielli, J. Sommeria, Excitation and breaking of internal gravity waves by parametric instability, J. Fluid Mech. 374 (1998) $117-144$.

[13] T. Peacock, P. Weidman, The effect of rotation on conical wave beams in a stratified fluid, Exp. Fluids 39 (2005) 32-37.

[14] N.H. Thomas, T.N. Stevenson, A similarity solution for viscous internal waves, J. Fluid Mech. 54 (1972) 495-506.

[15] D.G. Hurley, G. Keady, The generation of internal waves by vibrating elliptic cylinders. Part 2. Approximate viscous solution, J. Fluid Mech. 351 (1997) 119-138.

[16] L. Gostiaux, Etude expérimentale des ondes de gravité internes en présence de topographie. Emission, propagation, réflexion, Thèse de Doctorat de l'Ecole Normale Supérieure de Lyon, 2006. 
[17] B. Voisin, Internal waves from oscillating objects, in: Sixth International Symposium on Stratified Flows, Perth, Australia, 11-14 December 2006.

[18] B. Voisin, Added mass effects on internal wave generation, in: Fifth International Symposium on Environmental Hydraulics, Tempe, AZ, USA, 4-7 December 2007.

[19] C. Koudella, C. Staquet, Instability mechanisms of a two-dimensional progressive internal gravity wave, J. Fluid Mech. 548 (2006) 165-195.

[20] P.N. Lombard, J.J. Riley, Instability and breakdown of internal gravity waves. I. Linear stability analysis, Phys. Fluids 8 (12) (1996) 3271.

[21] M. Lesieur, Turbulence in Fluids, third ed., Kluwer Academic Publishers, 1997.

[22] G. Holloway, D. Ramsden, Theories of internal wave interaction and stably stratified turbulence: testing against numerical simulations, in: J.C. Nihoul, B.M. Jamard (Eds.), Small-Scale Turbulence and Mixing in the Ocean, Aha Huliko'a Hawaiian Winter Workshop, Elsevier, New York, 1988, pp. 363-377.

[23] W.H. Munk, A survey of internal waves and small scale processes, in: B.A. Warren, C. Wunsch (Eds.), Evolution of Physical Oceanography, MIT Press, Cambridge, MA, 1981, pp. 264-291.

[24] D. Fritts, M.J. Alexander, Gravity wave dynamics and effects in the middle atmosphere, Rev. Geophys. 41 (1) (2003), Art. No. 1003.

[25] M.L. Waite, P. Bartello, Stratified turbulence generated by internal gravity waves, J. Fluid Mech. 546 (2006) 313-339.

[26] C. Staquet, J.J. Riley, On the velocity field associated with potential vorticity, Dynam. Atmos. Oceans 14 (1989) 93-123.

[27] J.J. Riley, M.P. Lelong, Fluid motions in the presence of strong stable stratification, Annu. Rev. Fluid Mech. 32 (2000) 613-657.

[28] C. Cambon, Turbulence and vortex structures in rotating and stratified flows, Eur. J. Mech. B/Fluids 20 (2001) 489-510.

[29] C. Staquet, Gravity and inertia-gravity internal waves: breaking processes and induced mixing, Surv. Geophys. 25 (3-4) (2004) 281-314

[30] J.J. Riley, S.M. de Bruyn Kops, Dynamics of turbulence strongly influenced by buoyancy, Phys. Fluids 15 (2003) $2047-2059$.

[31] A.M. Fincham, T. Maxworthy, G.R. Spedding, Energy dissipation and vortex structure in freely-decaying stratified grid-turbulence, Dynam. Atmos. Oceans 23 (1996) 155-169.

[32] O. Praud, J. Sommeria, A.M. Fincham, Decaying grid turbulence in a rotating stratified fluid, J. Fluid Mech. 547 (2006) $389-412$.

[33] M. Gregg, Diapycnal mixing in the thermocline: a review, J. Geophys. Res. 92 (C5) (1987) 5249-5286.

[34] I.A. Hannoun, E.J. List, Turbulent mixing at a shear-free density interface, J. Fluid Mech. 189 (1988) 211-234.

[35] P. Bouruet-Aubertot, J. Sommeria, C. Staquet, Stratified turbulence produced by internal wave breaking: two-dimensional numerical experiments, Dynam. Atmos. Oceans 23 (1996) 357.

[36] A. Tabaei, T.R. Akylas, Nonlinear internal gravity wave beams, J. Fluid Mech. 482 (2003) 141-161.

[37] W. Munk, C. Wunsch, Abyssal recipes II: energetics of tidal mixing and wind mixing, Deep Sea Res. I 45 (1998) $1977-2010$.

[38] K.G. Lamb, Nonlinear interaction among internal wave beams generated by tidal flow over supercritical topography, Geophys. Res. Lett. 31 (2004) L09313.

[39] S. Legg, K.M.H. Huijts, Preliminary simulations of internal waves and mixing generated by finite amplitude tidal flow over isolated topography, Deep Sea Res. II 53 (2006) 140-156.

[40] T. Gerkema, C. Staquet, P. Bouruet-Aubertot, Nonlinear effects in internal tide beams and mixing, Ocean Modelling 12 (2006) $302-318$.

[41] T. Gerkema, C. Staquet, P. Bouruet-Aubertot, Decay of semi-diurnal internal-tide beams due to subharmonic resonance, Geophys. Res. Lett. 33 (2006) L08604.

[42] T. Hibiya, M. Nagasawa, Y. Niwa, Nonlinear energy transfer within the oceanic internal wave spectrum at mid and high latitudes, J. Geophys. Res. 107 (C11) (2002) 3207.

[43] T. Hibiya, M. Nagasawa, Y. Niwa, Global mapping of diapycnal diffusivity in the deep ocean based on the results of expendable current profiler (XCP) surveys, Geophys. Res. Lett. 33 (3) (2006) L03611.

[44] T. Gerkema, Internal and interfacial tides: beam scattering and local generation of solitary waves, J. Mar. Res. 59 (2) (2006) $227-255$.

[45] A.L. New, R.D. Pingree, Local generation of internal soliton packets in the central Bay of Biscay, Deep-Sea Res. 39 (1992) $1521-1534$.

[46] R.K. Zeytounian, Joseph Boussinesq and his approximation: a contemporary view, C. R. Mécanique 331 (2003) $575-586$.

[47] K. Ball, Energy transfer between external and internal gravity waves, J. Fluid Mech. 19 (1964) 465-478. 